\title{
SURTO DE MACRACANTORRINCOSE EM QUEIXADA (Tayassu pecari) CRIADO EXTENSIVAMENTE NOS ARREDORES DE GOIÂNIA - ESTADO DE GOIÁS, BRASIL: UMA ZOONOSE EM POTENCIAL
}

\author{
Marcos de Almeida Souza, ${ }^{1}$ Jurij Sobestiansky, ${ }^{1}$ Guido Fontgalland Coelho \\ Linhares, ${ }^{1}$ Willian P. de Oliveira ${ }^{2}$ e José Luiz de Barros Araújo ${ }^{3}$
}

\section{RESUMO}

\begin{abstract}
A macracantorrincose é considerada uma zoonose e, em virtude especialmente das características do sistema de criação, é praticamente inexistente na suinocultura moderna. Este trabalho tem como objetivo comunicar a ocorrência e as alterações anatomopatológicas de um surto de macracantorrincose causada por Macracanthorhynchus hirudinaceus em uma criação comercial de queixadas, Tayassu pecari. A criação, localizada nos arredores da cidade de Goiânia, Goiás, devidamente registrada no Instituto Brasileiro do Meio Ambiente e Recursos Naturais Renováveis, era composta por 150 animais de diferentes faixas etárias, os quais ocupavam uma área de 4,6 hectares, cercada por tela e com um curral de manejo em um dos piquetes. Além do pastejo os animais eram alimentados com milho e mandioca. No plantel houve mortalidade de 15 animais com idade inferior a 1 ano. Cinco destes animais foram necropsiados e, em seguida, foi realizada a colheita de amostras para exame microscópico e parasitológico. Na necropsia foram observadas: intensa deposição de fibrina sobre as vísceras abdominais, presença de líquido avermelhado na cavidade abdominal e extensa área de hemorragia e necrose no epíplon. A parede externa do intestino delgado apresentava inúmeros nódulos avermelhados, que à luz intestinal evidenciavam a presença de parasitos com corpo achatado e a probóscide aderida à mucosa intestinal. No exame histopatológico dos nódulos intestinais, verificou-se um infiltrado inflamatório granulomatoso acentuado, com fibroplasia moderada associada à presença de ovos, de larvas e da porção anterior do parasito. $\mathrm{O}$ exame parasitológico identificou o parasito como Macracanthorhynchus hirudinaceus, confirmando-se o surto de macracantorrincose.
\end{abstract}

DESCRITORES: Tayassuidae. Queixadas. Tayassu pecari. Macracanthorhynchus hirudinaceus. Enterite granulomatosa.

1 Professor do Departamento de Medicina Veterinária Preventiva. Escola de Veterinária (EA), Universidade Federal de Goiás (UFG).

2 Médico Veterinário Autônomo.

3 Professor de Instituto de Patologia Tropical e Saúde Pública (IPTSP).

Endereço para correspondência: Escola de Veterinária, Campus II, Cx Postal 131, CEP 74001-970, Goiânia, Goiás. Fax: 3521-1597; E-mail: souzavet@pop.com.br

Recebido para publicação em 24/10/2005. Revisto em 19/1/2006. Aceito em 31/1/2006. 


\section{INTRODUÇÃO}

Há uma crescente demanda mundial por carnes de animais selvagens e exóticos por apresentarem sabor diferenciado e serem consideradas mais saudáveis. Produtos como carne de javali Sus scrofa Linnaeus, 1758, de queixada Tayassu pecari (Link, 1795; Fisher, 1817) e de cateto Pecari tajacu (Linneaus, 1758; Reichenbach, 1815) estão em ascensão no mercado brasileiro. O queixada é um mamífero que em seu estado natural vive em bandos, alimenta-se basicamente de raízes, frutos, larvas, vermes e até mesmo de cobras. Se capturado jovem, é facilmente domesticado, podendo ser alimentado com ração utilizada para suínos e/ou alimentos equivalentes, tais como: milho, batata-doce, capim, talos de verdura e até cascas de banana $(16,17)$.

A observação e o estudo das patologias dos animais possibilitam compreender as possíveis relações com o ser humano. Com base nesta observação molda-se a saúde pública e assumem importância as enfermidades que são transmitidas dos animais para o homem (11).

A macracantorrincose animal é uma endoparasitose zoonótica. Há relatos de macracantorrincose humana, cujo quadro clínico é similar ao que ocorre nos animais. Esta parasitose tem sido registrada nos seguintes países: Tailândia (15), China (9), Papua Nova Guiné (13), Estados Unidos da América (5), Republica Tcheca e Perú (3). No Brasil esta zoonose foi registrada no Rio Grande do Norte (7).

A principal espécie que acomete os suídeos e afins é o Macracanthorhynchus hirudinaceus. Esteparasitoé caracterizado pela fixação na parede do intestino delgado por uma probóscide formada por anéis espinhosos, gerando nódulos também denominados "marcas de amora". Ao provocar uma enterite granulomatosa, pode ocorrer uma ruptura da parede, desencadear uma peritonite e ocasionar a morte dos indivíduos (8). A transmissão ocorre pela ingestão do hospedeiro intermediário, os coleópteros coprófagos da família Scarabeidae, portadores de larvas infectantes $(6,11)$.

$\mathrm{O}$ presente relato tem como objetivo descrever a ocorrência de um surto de macracantorrincose animal provocada por Macracanthorhynchus hirudinaceus em uma criação comercial de queixadas nos arredores da cidade de Goiânia, no estado de Goiás.

\section{MATERIAL E MÉTODOS}

A criação de queixada, Tayassu pecari, localizada nos arredores da cidade de Goiânia (GO), devidamente registrada no Instituto Brasileiro do Meio Ambiente e Recursos Naturais Renováveis (IBAMA), era composta por 150 animais de diferentes faixas etárias, os quais ocupavam uma área de 4,6 hectares, dividida em três piquetes, cercada por tela de 1,60 m de altura. Em um dos piquetes existia uma área cercada que servia como um pequeno curral destinado ao manejo dos animais (Figura 1). Além do pastejo os animais eram alimentados com milho e mandioca fornecidos 
diariamente no mesmo horário. No plantel houve 15 mortes de animais de várias faixas etárias no período de um ano. A criação foi visitada e os animais inspecionados visualmente antes e após o arraçoamento. Não foi possível entrar no piquete por causa da agressividade dos animais, o que impossibilitou o exame individual.

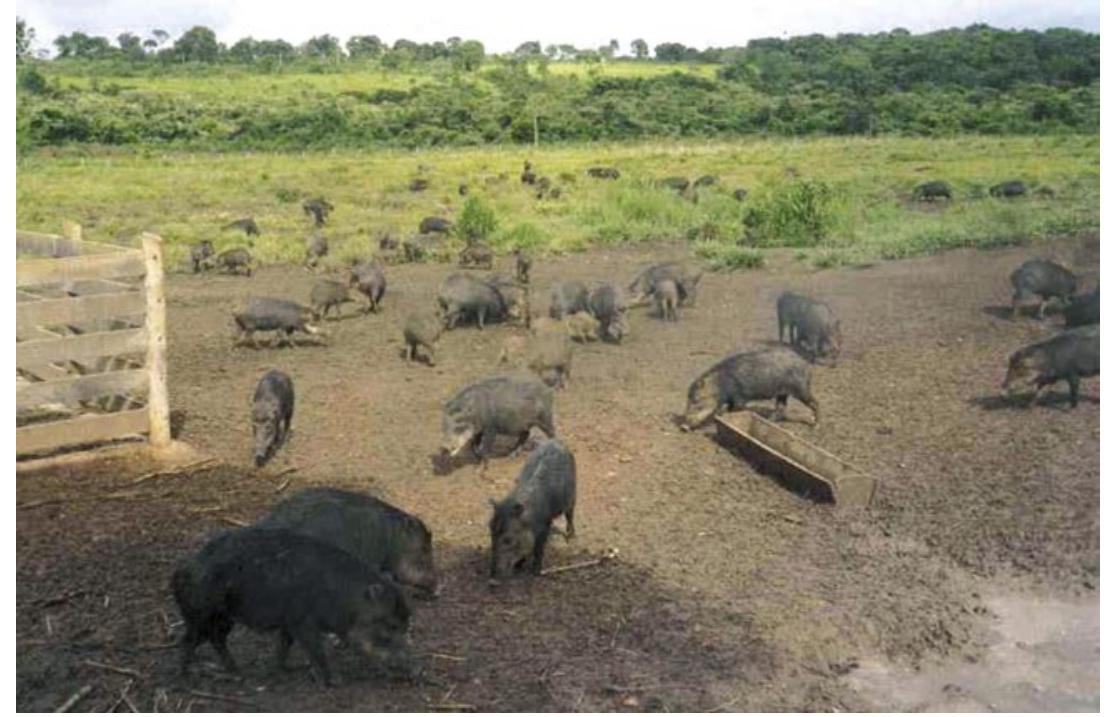

Figura 1. Piquete de manejo da criação de queixadas, Tayassu pecari, nos arredores de Goiânia, estado de Goiás

Segundo o médico veterinário responsável pela criação, os animais que vieram a óbito apresentaram sintomatologia clínica de isolamento dos demais, anorexia e apatia. Cinco destes animais foram encaminhados ao Setor de Patologia Animal da Escola de Veterinária - UFG (Registro de Necropsia - Janeiro 2003, Caderno 30, Nos 844, 845 e 846), os quais foram necropsiados seguindo-se a técnica descrita anteriormente. As amostras de tecidos (intestino delgado, intestino grosso, estômago, fígado, coração, pulmão e rins) foram conservadas em formol tamponado a $10 \%$ para exame microscópico (18). As amostras de parasitos foram acondicionadas em formol tamponado a $10 \%$; as fezes foram colhidas do reto dos animais necropsiados para o exame parasitológico pela técnica de sedimentação e enviadas para o Laboratório de Doenças Parasitárias da Escola de Veterinária da UFG. No laboratório, os parasitos foram clarificados com ácido glacial acético e montados em lâminas de microscópio para a identificação morfológica, de acordo com as características taxonômicas descritas $(12,14)$. Para o exame histológico, as amostras de tecidos foram desidratadas, diafanizadas e incluídas em parafina (10). Os cortes com espessura de $4 \mu \mathrm{m}$ foram corados com hematoxilina e eosina e examinados em microscópio óptico de campo claro. 


\section{RESULTADOS E DISCUSSÃO}

A macracantorrincose usualmente é assintomática (19). Este fato vem ao encontro do relato do tratador, pois, segundo ele, na maioria dos casos não foram observados sintomas e os animais, simplesmente, foram encontrados mortos no piquete. No entanto, em um ou outro caso ele observou que, num período de um a dois dias que antecederam a morte, os animais não se alimentavam, deslocavam-se lentamente e procuravam afastar-se do grupo, permanecendo grande parte do tempo deitados, quando possível na água.

Por ocasião da necropsia dos animais foi possível observar intensa deposição de fibrina sobre as vísceras abdominais, cerca de 250 a $300 \mathrm{~mL}$ de um líquido avermelhado na cavidade abdominal, e extensa área de hemorragia e necrose no epíplon. Na parede do intestino delgado havia numerosos nódulos de coloração avermelhada a partir da região serosa (Figuras 2 e 3), com uma média de 90 nódulos/ animal, medindo aproximadamente 0,5 a 1,0 cm de diâmetro. Esses nódulos são considerados próprios da macracantorrincose $(3,19)$. Após a abertura do intestino delgado, observou-se a presença de parasitos de corpo achatado e a probóscide aderida à mucosa intestinal (Figura 4).

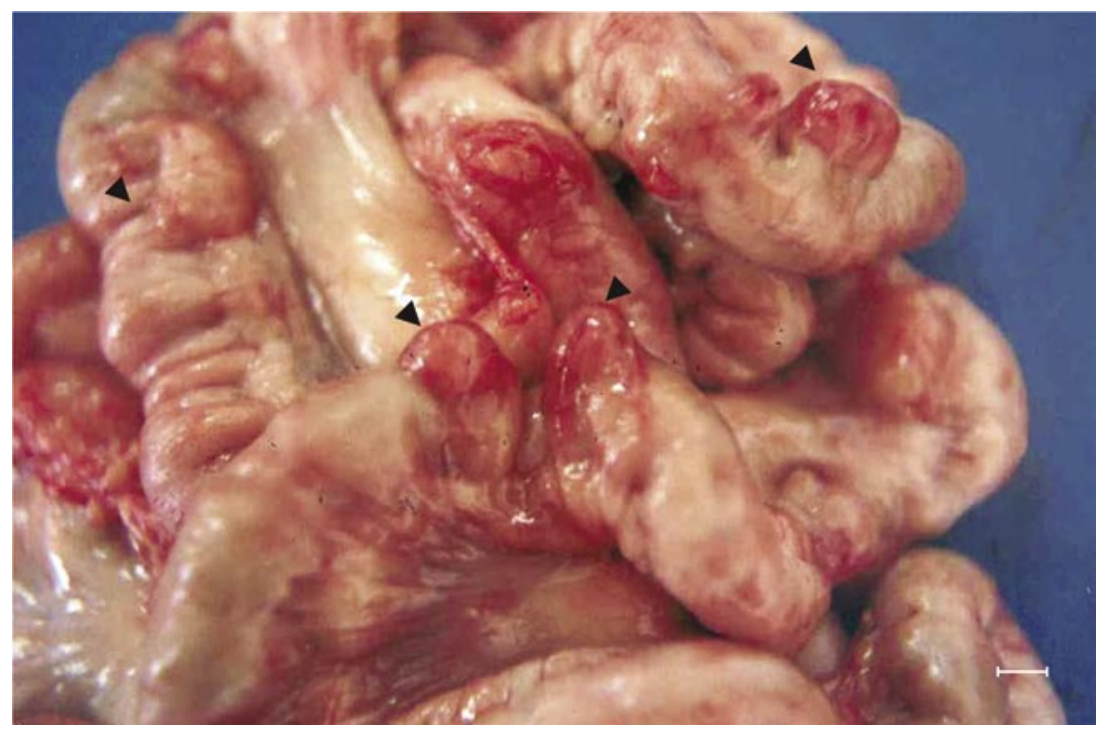

Figura 2. Intestino delgado de Tayasssu pecari. Setas indicam os nódulos avermelhados na região serosa, também denominados "manchas de amora", características da macracantorrincose $($ Barra $=1 \mathrm{~cm})$.

No exame histopatológico, somente foram verificadas alterações nos nódulos intestinais caracterizadas por um infiltrado inflamatório granulomatoso 
acentuado, com fibroplasia moderada associada à presença de ovos, de larvas e da porção anterior do parasito. Esses achados anatomopatológicos e histopatológicos são semelhantes aos descritos por outros autores $(2,3,4,8,19)$.

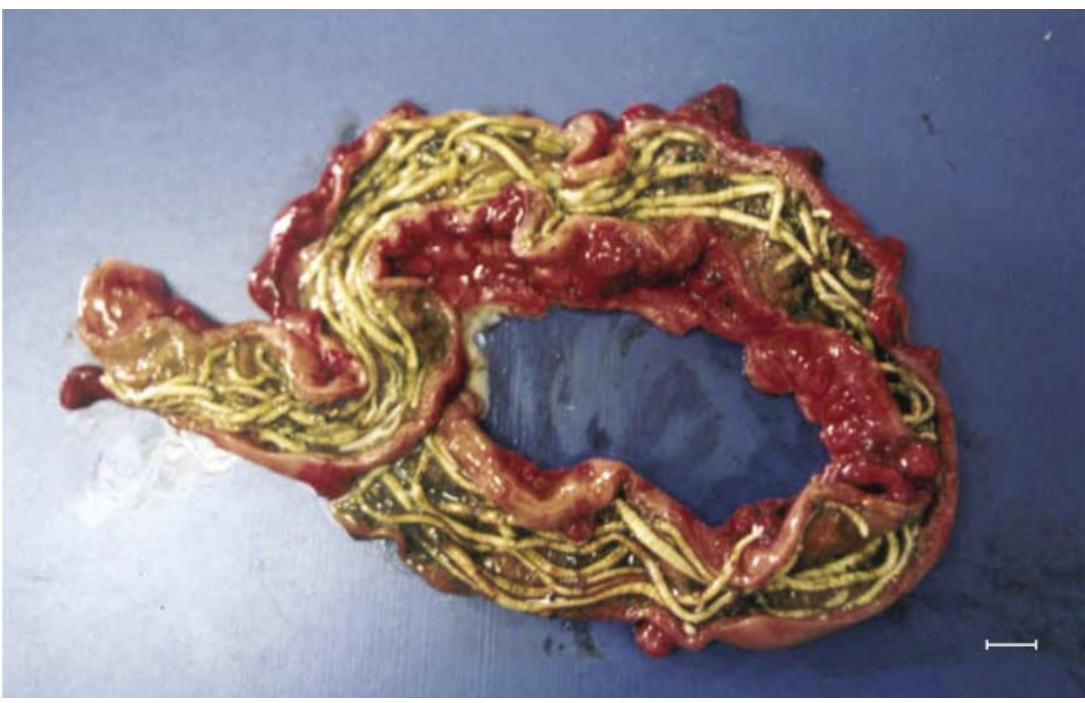

Figura 3. Intestino delgado de Tayasssu pecari repleto de Macracanthorhynchus hirudinaceus $($ Barra $=1 \mathrm{~cm})$.

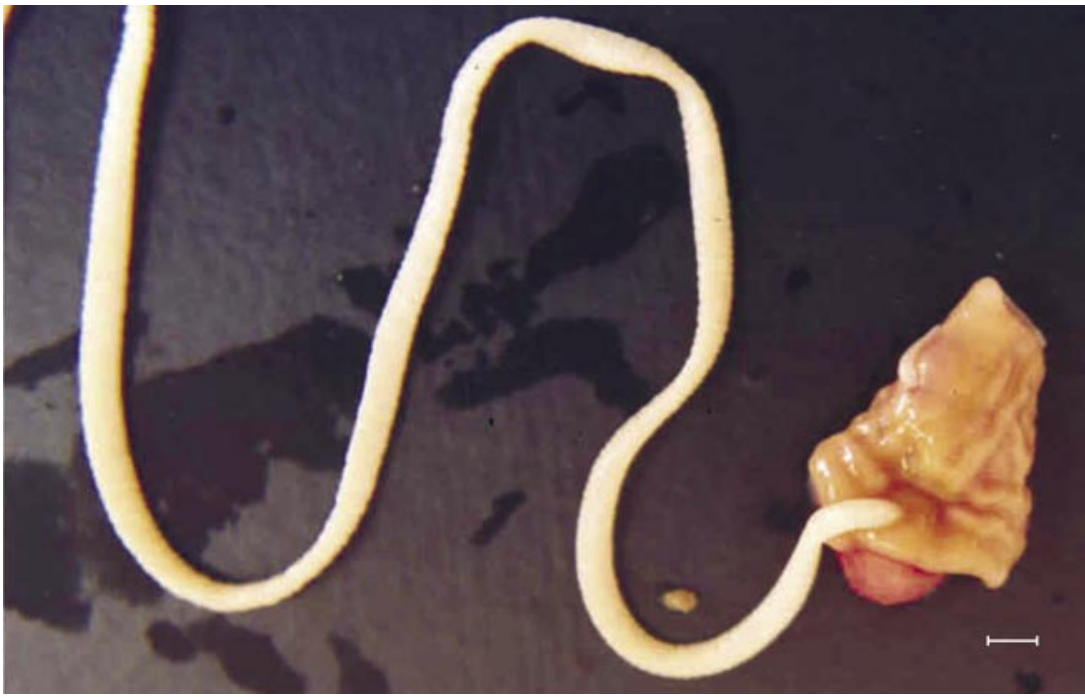

Figura 4. Macracanthorhynchus hirudinaceus aderido à mucosa intestinal de Tayassu pecari, formando o nódulo na região serosa (manchas de amora). 
No exame morfológico dos parasitos montados em lâminas, foram observados machos com o corpo levemente curvado ventralmente em forma de fita, pseudosegmentados, com comprimento de 5 a $10 \mathrm{~cm}$ e largura de $3 \mathrm{a} 5 \mathrm{~mm}$; probóscide com 0,5 a $0,8 \mathrm{~mm}$, globular, com 5 a 6 fileiras de ganchos de tamanhos decrescentes para a base, os menores com $260 \mu \mathrm{m}$ de espessura e os maiores com $400 \mu \mathrm{m}$ de comprimento; pescoço cônico, lemniscos claviformes com 15mm de comprimento; testículos volumosos, com o distal em contato com os lemniscos; glândulas prostáticas em número de oito dispostas duas a duas e extremidade posterior campanuliforme. As fêmeas eram achatadas, com corpo rugoso com 10 a $35 \mathrm{~cm}$; núcleos ovígeros dispersos na cavidade geral; vulva situada subterminalmente e ovos com 80 a $100 \mu \mathrm{m}$ de comprimento por 51 a $56 \mu \mathrm{m}$ de largura.

Em todas as amostras analisadas, o exame parasitológico das fezes apresentou grande número de ovos típicos da espécie $M$. hirudinaceus. A caracterização morfológica de parasitos adultos e ovos permitiu a identificação dos parasitos como M. hirudinaceus (Pallas, 1781; Travassos, 1917), segundo descrições taxonômicas propostas por outros autores $(12,14)$.

$\mathrm{O}$ grande número de helmintos adultos encontrados no intestino delgado dos animais necropsiados e a presença de lesões macroscópicas, como perfurações da parede intestinal e intensa deposição de fibrina no peritônio, caracterizam um quadro semelhante ao registrado por outros autores que observaram os aspectos da macracantorrincose $(1,6)$.

\section{CONCLUSÃO}

Este é o primeiro registro de Macracanthorhynchus hirudinaceus parasitando queixadas, Tayassu pecari, no Brasil.

Os resultados encontrados neste estudo servem de alerta tanto para os produtores interessados em criação de queixada em cativeiro quanto para a comunidade científica, na área da saúde pública, sobre ocorrência da enfermidade em animais e sua possível transmissão aos habitantes dos arredores da cidade de Goiânia.

\section{ABSTRACT}

Macracanthorhynchus hirudinaceus infection outbreak in white-lipped peccary, Tayassu pecari, raised extensively on the outskirts of Goiânia, State of Goiás, Brazil: a potential zoonosis

Macracanthorhynchus hirudinaceus infection which currently does not occur in modern swine production systems, mainly due to the raising characteristics, is considered a zoonosis. The purpose of this paper is to report the occurrence and the anatomopathological diagnosis of an outbreak of Macracanthorhynchus hirudinaceus infection in a commercial farm of white-lipped peccaries. At the farm, 
located on the outskirts of the city of Goiânia in the State of Goiás, and registered in the Instituto Brasileiro do Meio Ambiente e Recursos Naturais Renováveis , there were 150 animals of different ages, living in an area of 4.6 hectares, surrounded with wire screen fence and with a special paddock used for management. Besides foraging, the animals were fed with corn and cassava. In the stock, a mortality of 15 animals, less than one year old, was registered. Post-mortem examination was carried out on five of them, and samples were sent for microscopic and parasitological examination. Intense deposition of fibrin on abdominal organs, presence of reddish liquid in the abdominal cavity, large hemorrhagic area and necrosis of the epiploon were found in the post-mortem examination. In the small intestine wall there were many reddish nodules with flattened body parasites inside. These parasites had their proboscides attached to the intestinal mucosa. A granulomatous inflammatory infiltrate with moderate fibroplasia associated to the presence of eggs, larvae and proboscis of the adult parasite were found in the histopathological examination. During the parasitological examination the parasite was identified as Macracanthorhynchus hirudinaceus, confirming the outbreak.

KEYWORDS: Tayassuidae. White-lipped peccaries. Tayassu pecari. Macracanthorynchus hirudinaceus. Granulomatous enteritis .

\section{REFERÊNCIAS}

1. Acha PN, Szyfres B. Zoonosis y enfermedades transmisibles comunes al hombre y a los animales. 2. ed. Organización Panamericana de la Salud, Washington, 1986. 989 p.

2. Barker IK, Van Dreumel AA, Palmer N. The alimentary system. In: Pathology of Domestic Animals. Eds. Jubb KVF, Kenedy PC, Palmer N. 4.ed. San Diego, 1993. p. 294-295.

3. BeltránFdeEstradaM.Presentación del primercasohumanodeparasitismopor Macracanthrhynchus hirudinaceus en el Perú y breve revisión. Rev Peru Med Exp Salud Pública 14: 47-50.1997.

4. Corwin RM, Stewart TB. Internal Parasites. In: Disease of Swine. Eds. Leman AD, Straw BE, Mengeling WL, Allaire SD, Taylor DJ. 7ed. Iowa University Press, Ames, 1993. p. 713-734.

5. Dingley D, Beaver PC. Macracanthorhynchus ingens from a child in Texas. Am J Trop Med Hyg 34: 918-920, 1985.

6. Freitas MG. Helmintologia Veterinária. Belo Horizonte: Rabelo \& Brasil, 1977.

7. Gonzaga AG. Assumptos de Actualidade. Contribuição ao saneamento do Nordeste. Brasil Med 35:134-135, 1921.

8. Jones TC, Hunt RD, King NW. Veterinary Pathology. 6th ed. Williams \& Wilkins, Maryland, 1997. $1.392 \mathrm{p}$

9. Leng YJ, Huang WD, Liang PN. Human infection with Macracanthorhynchus hirudinaceus Travassos, 1916 in Guangdong Province, with notes on its prevalence in China. Ann Trop Med Parasitol 77: 107-109, 1983.

10. Luna LG. Manual of histologic staining methods of the Armed Forces Institute of Pathology. 3. ed. New York, 1968. 258 p.

11. Milhaud C. Zoonoses et maladies transmissibles communes a l'homme et aux animaux: point de vue vétérinaire. Rev Fr Lab 310: 77-94, 1999.

12. Neveu-Lemaire M. Traité d'helmithologie médicale et vétérinaire. Vigot Frères, Paris, 1936. 1514 p.

13. Owen I L. Parasitic zoonoses in Papua New Guinea. J Helminthol 79: 1-14, 2005.

14. Pinto C. Zooparasitos de interesse médico e veterinário. Pimenta de Melo, Rio de Janeiro, 1945.376 p. 
15. Radomyos P, ChobchuanchomA, TungtrongchitrA. Intestinal perforation due to Macracanthorhynchus hirudinaceus infection in Thailand. Trop Med Parasitol 40: 476-467, 1989.

16. Raynaud JP. Les parasites de porc. Que sont-ils? Que font-ils? Aspects economiques actualisés de la prevention parasitaire. $L^{`}$ Eleveur 163: 75-80, 1984.

17. Silva CAB, Batalha MO. Competitividade em Sistemas Agroalimentares. Ribeirão Preto, 1999. p. 9-20.

18. Silveira D, Sobestiansky J, Vieira RP. Técnica de necropsia em suínos. In: Manual de práticas veterinárias. Lisboa: Fundação Calouste Gulbenkian, 2002. 366 p.

19. Sobestiansky J, Barcellos DESN, Mores N, Oliveira SJ, Carvalho LFOS, Moreno AM, Roehe PM. Clínica e patologia suína. Goiânia, 1999. p. 271-273. 